Bull. Astr. Soc. India (2011) 39,1-??

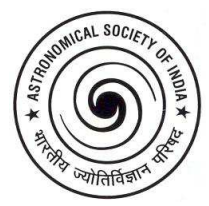

\title{
Early Universe with CMB polarization
}

\author{
Tarun Souradeep* \\ IUCAA, Post Bag 4, Ganeshkhind, Pune, India
}

Received 2011 April 14; accepted 2011 April 15

\begin{abstract}
The Universe is the grandest conceivable scale on which the human mind can strive to understand nature. The amazing aspect of cosmology, the branch of science that attempts to understand the origin and evolution of the Universe, is that it is largely comprehensible by applying the same basic laws of physics that we use for other branches of physics. The observed cosmic microwave background (CMB) is understood by applying the basic laws of radiative processes and transfer, masterfully covered in the classic text by S. Chandrasekhar, in the cosmological context. In addition to the now widely acclaimed temperature anisotropy, there is also linear polarization information imprinted on the observed Cosmic Microwave background. CMB polarization already has addressed, and promises to do a lot more, to unravel the deepest fundamental queries about physics operating close to the origin of the Universe.
\end{abstract}

Keywords : cosmic microwave background - early Universe - polarization - radiative transfer

\section{Introduction}

It is an honour to write an invited article commemorating the birth centenary of Nobel laureate, Professor Subrahmanyan Chandrasekhar. The Universe is the grandest conceivable scale on which the human mind can strive to understand nature. Remarkably, even the origin and evolution of the Universe is largely comprehensible by applying the same basic laws of physics that are used in many other branches of physics. Chandrasekhar's research epitomizes this amazing reality, that one can understand complex phenomena in astrophysics by building theories based on the basic laws of physics. This article is devoted to the cosmic microwave background (CMB), in particular, the measured intensity and polarization fluctuations. The physics of this emerging champion among cosmological observables is based on straightforward application of the theory of radiative transfer of the relic radiation from big bang through the cosmic eons - a subject

*e-mail:tarun@iucaa.ernet.in 
that has been masterfully enshrined in the classic text 'Radiative transfer' of S. Chandrasekhar (1960). This text is, in fact, cited in the seminal papers on CMB anisotropy and polarization and, subsequent reviews (Peebles \& Yu 1970; Bond \& Efstathiou 1984, 1987; Bond 1996).

Historically, theoretical development always preceded observations in cosmology up until the past couple of decades. However, in sharp contrast, recent developments in cosmology have been largely driven by huge improvements in quality, quantity and the scope of cosmological observations. There are two distinct aspects to modern day cosmology - the background Universe and the perturbed Universe. The 'standard' model of cosmology must not only explain the dynamics of the homogeneous background Universe, but also satisfactorily describe the perturbed Universe - the generation, evolution and finally, the formation of the large-scale structure (LSS) in the Universe observed in the vast galaxy surveys. It is fair to say that cosmology over the past few decades has increasingly seen intense interplay between the theory and observations of the perturbed Universe. Spectacular breakthroughs in various observations have now concretely verified that the present edifice of the standard cosmological models is robust. A set of foundations and pillars of cosmology have emerged and are each supported by a number of distinct observations, which are listed below.

- Homogeneous, isotropic Universe, expanding from a hot initial phase due to gravitational dynamics described by the Friedman equations derived from laws of General Relativity.

- The basics constituents of the Universe are baryons, photons, neutrinos, dark matter and dark energy (cosmological constant/vacuum energy).

- The homogeneous spatial sections of space-time are nearly geometrically flat (Euclidean space).

- Evolution of density perturbations under gravitational instability has produced the largescale structure in the distribution of matter starting from the primordial perturbations in the early Universe.

- It has been established that the primordial perturbations have correlation on length scales larger than the causal horizon; this makes a strong case for an epoch of inflation in the very early Universe. The nature of primordial perturbations match that expected from the generation of primordial perturbations in simplest models of inflation.

The cosmic microwave background, a nearly uniform, thermal black-body distribution of photons throughout space, at a temperature of 2.7 degrees Kelvin, accounts for almost the entire radiation energy density in the Universe. Tiny variations of temperature and linear polarization of these black-body photons of the cosmic microwave background arriving from different directions in the sky faithfully encode information about the early Universe. Further these photons have travelled unimpeded across the entire observable Universe making them excellent probes of the Universe on the largest observable scales. The much talked about 'dawn of precision era of cosmology' has been ushered in by the study of the perturbed Universe. Measurements of CMB anisotropy and polarization have been by far the most influential of the cosmological observations driving advances in current cosmology in this direction. 


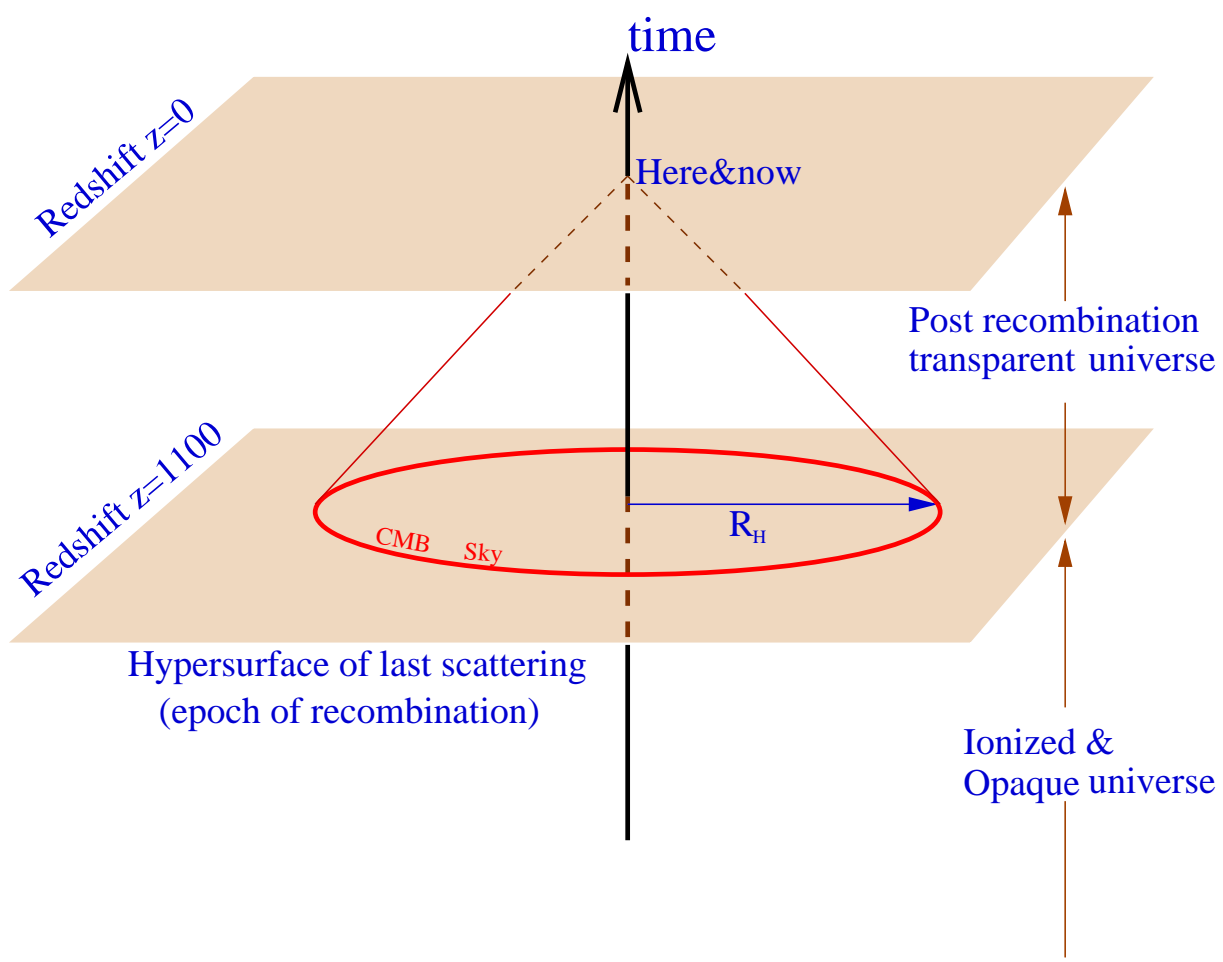

Figure 1. A cartoon explaining the Cosmic Microwave Background (CMB) using a space-(conformal) time diagram. The present Universe is transparent and CMB photons travel to us freely over cosmic distances along our past light cone. In an expanding Universe, the temperature of the Planck black-body CMB is inversely proportional to the expansion factor. When the Universe is about 1100 times smaller, the CMB photons are just hot enough to keep the baryonic matter in the Universe (about 3 quarters Hydrogen, 1 quarter Helium as determined by big bang nucleosynthesis) ionized, and at that epoch there is a sharp transition to an opaque Universe in the past. The CMB photons come to us unimpeded directly from this spherical opaque surface of last scattering at a distance of $R_{H}=14 \mathrm{Gpc}$ that surrounds us - a super IMAX cosmic screen. The red circle depicts the sphere of last scattering in the reduced $2+1$ dimensional representation of the Universe.

\section{CMB anisotropy and polarization}

The CMB photons arriving from different directions in the sky show tiny variations in temperature, at a level of ten parts per million, i.e., tens of micro-Kelvin, referred to as the CMB anisotropy, and a net linear polarization pattern at micro-Kelvin to tens of nano-Kelvin level. The tiny variations of temperature and linear polarization of these black-body photons of the cosmic microwave background arriving from different directions in the sky have freely propagated over cosmological distances and carry information of the the early Universe. As illustrated in the cartoon in Figure 1, the cosmic microwave background radiation sky is essentially a giant, cosmic 
'super' IMAX theater screen surrounding us at a distance of 14 billion parsecs displaying a snapshot of the Universe at a time very close to its origin. Hence the CMB anisotropy and polarization are imprints of the perturbed Universe in the radiation when the Universe was only 0.3 millions years old, compared to its present age of about 14 billion years.

It is convenient to express the sky map of CMB temperature anisotropy, $\Delta T(\hat{\mathbf{n}})$ (and polarization, as we shall discuss later) in the direction $\hat{\mathbf{n}}$ in a spherical harmonic expansion :

$$
\Delta T(\hat{\mathbf{n}})=\sum_{\ell=2}^{\infty} \sum_{m=-\ell}^{\ell} a_{\ell m} Y_{\ell m}(\hat{\mathbf{n}}) .
$$

Theory predicts that the primary CMB anisotropy is a statistically isotropic, Gaussian field (of zero mean), and current observations remain fully consistent with this expectation. The anisotropy can then be characterized solely in terms an angular power spectrum

$$
C_{\ell}=\frac{1}{(2 \ell+1)} \sum_{m=-\ell}^{\ell}\left|a_{\ell m}\right|^{2} .
$$

The $C_{\ell}$ spectra for a wide range of parameters within the 'standard' cosmology share a generic set of features neatly related to basics physics, governing the CMB photon distribution function. On the large angular scales (low multipole, $\ell$ ), the CMB anisotropy directly probes the primordial power spectrum of metric fluctuations (scalar gravitational potential and tensor gravitational waves) on scales enormously larger than the 'causal horizon'. On smaller angular scales $(150<\ell<1500)$, the CMB temperature fluctuations probe the physics of the coupled baryonphoton fluid through the imprint of the acoustic oscillations in the ionized plasma sourced by the same primordial fluctuations. At even higher multipoles, the damping tail of the oscillations encodes interesting physics such as the slippage in the baryon-photon coupling, temporal width of the opaque to transparent Universe transition, weak lensing due to large scale structures in the Universe. Fig. 2 that dissects the CMB angular power spectrum attempts to provide a cryptic summary of the various kinds of physics involved. Overall, the physics of CMB anisotropy has been very well understood for more than two decades, Furthermore, the predictions of the primary anisotropy and linear polarization and their connection to observables are, by and large, unambiguous (Bond 1996; Hu \& Dodelson 2002).

The acoustic peaks occur because the cosmological perturbations excite acoustic waves in the relativistic plasma in the early Universe. The recombination of baryons at redshift $z \approx 1100$ effectively decouples the baryon and photons in the plasma abruptly switching off the wave propagation. In the time between the excitation of the perturbations and the epoch of recombination, modes of different wavelength can complete different numbers of oscillation periods, or in other words, waves can travel a finite distance and then freeze. This translates the characteristic time scale into a characteristic length scale and leads to a harmonic series of maxima and minima in the CMB anisotropy power spectrum. The acoustic oscillations have a characteristic scale known 


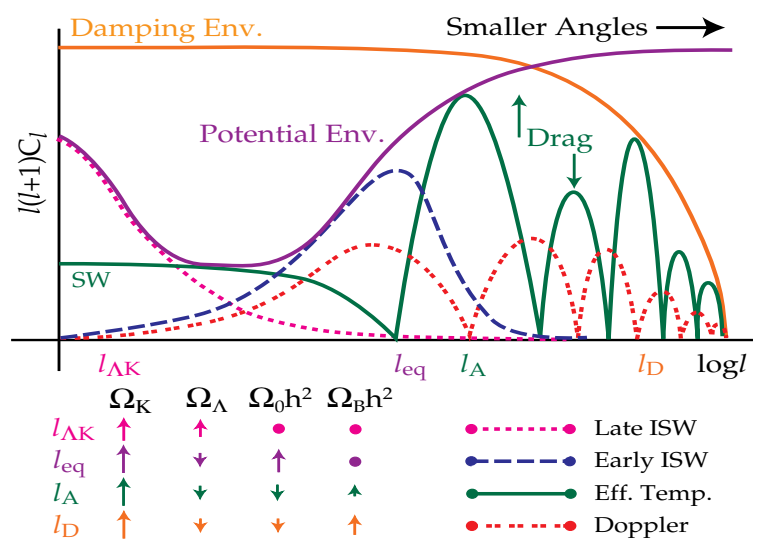

Figure 2. Figure taken from $\mathrm{Hu}$, Sugiyama \& Silk (1997) summarizes the different contributions to the primary CMB anisotropy. Also indicated is the dependence of the four length scales that are imprinted on the $C_{\ell}$ spectrum on some of the cosmological parameters. The Sachs-Wolfe (SW) plateau at low $\ell$ is a faithful reproduction of the near scale-invariant spectrum of initial metric perturbations. Integrated SachsWolfe (ISW) effect arises from the evolution of metric perturbations along the path of free streaming CMB photons. Late ISW arises on $\ell<\ell_{\Lambda K}$ if the Universe has significant curvature or cosmological constant. The early ISW contribution at $\ell \sim \ell_{\mathrm{eq}}$ is due to transition from radiation to matter domination. The acoustic and Doppler terms give rise to a harmonic series of oscillatory peaks as a snapshot of the oscillations of a viscous baryon-photon fluid prior to the epoch of recombination. The sound horizon at recombination sets the length scale of the acoustic oscillations. This 'standard ruler' at $z \approx 1100$ then allows an accurate determination of the geometry of the Universe from the location of the first peak, $\ell_{A}$ via the angle-distance relationship. High baryon density increases viscous drag leading to suppression of even numbered acoustic peaks relative to odd. Power is exponentially damped at large $\ell$ due to photon diffusion out of matter over-densities (Silk damping) and finite thickness of the last scattering surface.

as the sound horizon, which is the comoving distance that a sound wave could have traveled up to the epoch of recombination. This well-determined physical scale of $150 \mathrm{Mpc}$ is imprinted on the CMB fluctuations at the surface of last scattering, the typical scale of the random bright and dull patches on the 'cosmic super-IMAX' screen.

The angle subtended by this physical scale in the CMB sky (IMAX screen) at a known distance of $14 \mathrm{Gpc}$ then allows a sensitive determination of the geometry $\left(\Omega_{0 K}\right)$ of the background Universe. Essentially, the same standard ruler of $150 \mathrm{Mpc}$ placed at $14 \mathrm{Gpc}$ would subtend different angles in a Universe with different spatial curvature. This determines the location of the series of harmonic peaks of $C_{\ell}$ along the multipole $\ell$ seen in Fig. 2. The amplitude of baryon-photon oscillations can be expected to directly scale with the amount of baryons available in the Universe. Consequently, the height of the peaks in the $C_{\ell}$ sensitively determine the baryon density, $\Omega_{B}$. The $C_{\ell}$ s are sensitive to other important cosmological parameters, such as, the relative den- 

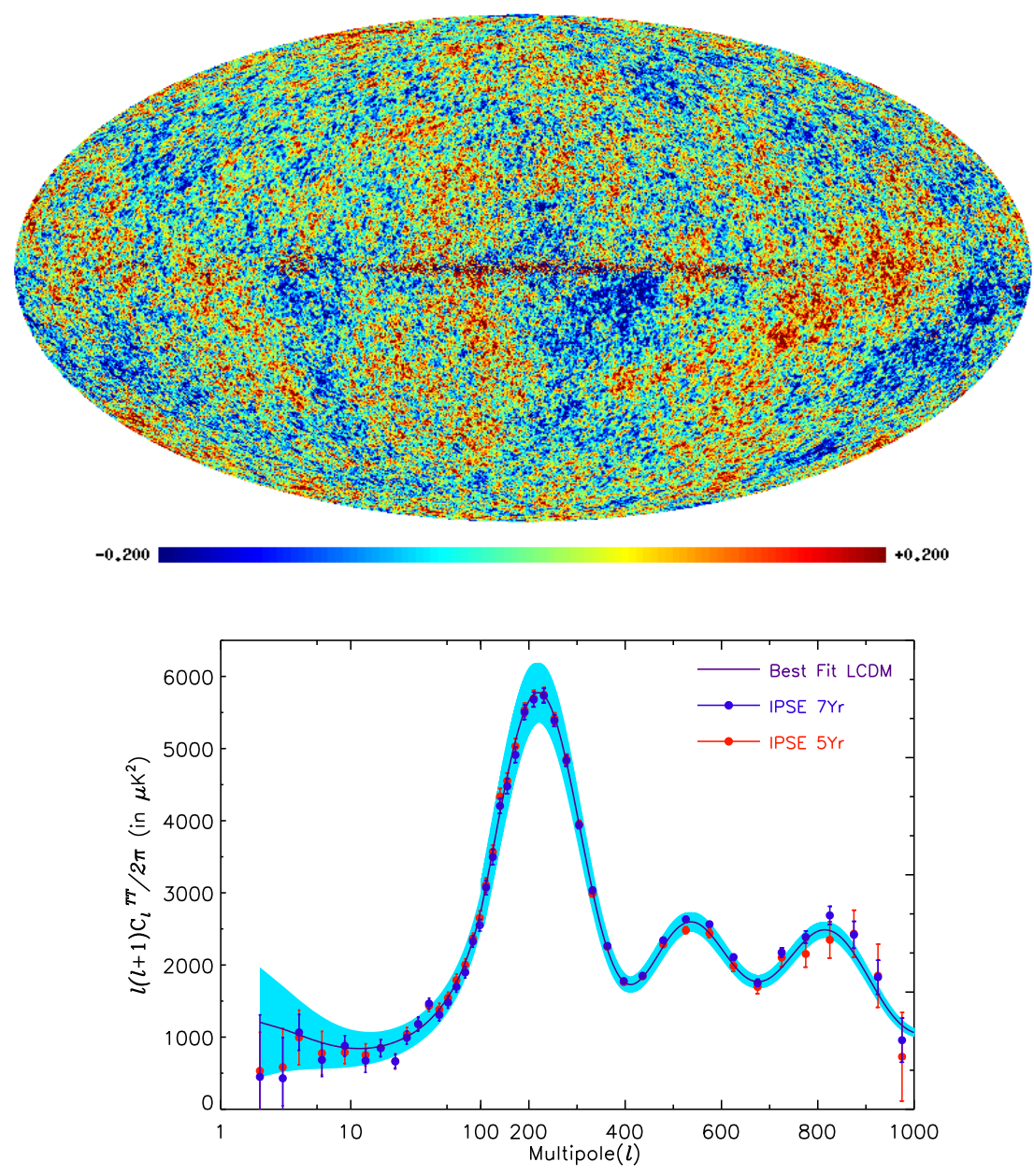

Figure 3. The exquisite temperature anisotropy data from the three years of data from the WMAP satellite is shown in the figures. Top: The top figure shows colour-coded full sky map (in Mollewide projection) of the CMB temperature variations. The temperature variations range between $\pm 200 \mu \mathrm{K}$ with an rms of about $70 \mu \mathrm{K}$. The angular resolution of features of the map is about a quarter of a degree. For comparison, the first CMB anisotropy measurements in 1992 by the DMR instrument on board the COBE satellite produced the same map at a much coarser resolution of 7 degrees. Bottom: The angular power spectrum estimated from the multi-frequency five- and seven-year WMAP data. The result from IPSE, a self-contained model free approach to foreground removal (Saha, Jain \& Souradeep 2006; Samal et al. 2010) matches that obtained by the WMAP team. The solid curve showing prediction of the best fit power-law, flat, $\Lambda$ CDM model threads the data points closely [Figure: courtesy Tuhin Ghosh]. 
sity of matter, $\Omega_{m}$, cosmological constant, $\Omega_{\Lambda}$, Hubble constant, $H_{0}$ and deviation from flatness (curvature), $\Omega_{K}$. Implicit in $C_{\ell}$ is the hypothesized nature of random primordial/initial metric perturbations - (Gaussian) statistics, (nearly scale-invariant) power spectrum, (largely) adiabatic vs. iso-curvature and (largely) scalar vs. tensor component. The 'default' settings in bracket are motivated by inflation (Starobinsky 1982; Guth \& Pi 1982; Bardeen, Steinhardt \& Turner 1983).

Besides, the entirely theoretical motivation of the paradigm of inflation, the assumption of Gaussian, random adiabatic scalar perturbations with a nearly scale-invariant power spectrum is arguably also the simplest possible theoretical choice for the initial perturbations. What has been truly remarkable is the extent to which recent cosmological observations have been consistent with and, in certain cases, even vindicated the simplest set of assumptions for the initial conditions for the perturbed Universe discussed below.

The first two decades ( 1991-2011) of exciting CMB anisotropy measurements have been capped off with the release of 7 years of data from the Wilkinson Microwave Anisotropy Probe (WMAP) of NASA 11. The first detection of CMB anisotropy by COBE-DMR in 1992 observationally established the origin and mechanism of structure formation in the Universe. Observations were then made at three frequencies, 90,53 and $31 \mathrm{GHz}$ which allowed a fairly good removal of the 'foreground' contamination of the cosmic signal by the strong emission from our own Galaxy. The 15-years old experimental success story of CMB anisotropy measurements, starting from discovery of CMB anisotropy by the COBE satellite in 1992, has been topped off by the exquisite data from the WMAP. The WMAP satellite was placed at the second Lagrange point of the Sun-Earth system. Measurements from WMAP combine high angular resolution with full sky coverage and high sensitivity due to the stable thermal environment allowed by a space mission. Moreover, observations were made at five frequencies, 94 (W-band), 61 (Vband), 41 (Q-band), 33 (Ka-band) and $23 \mathrm{GHz}$ (K-band) that allowed much better removal of the 'foreground' contamination. Similar to the observational strategy of COBE-DMR, the satellite measures CMB temperature differences between a pair of points in the sky. Each day the satellite covered $30 \%$ of the sky, but covers the full sky in 6 months. This massive redundancy in measurements allows the mission to beat down the detector noise to from milli-Kelvins to tens of micro-Kelvin level. The WMAP mission has acquired data for about nine years up until August 2010 and made that public at regular intervals after a short proprietary possession (first year data was released in 2003, three year data in 2006, five year data in 2008, and seven year data in 2010). A final data release of the entire nine years of data in expected in the coming year.

The measured angular power spectrum of the cosmic microwave background temperature fluctuations, $C_{\ell}$, shown in Fig. 3 has become invaluable for constraining cosmological models. The position and amplitude of the peaks and dips of the $C_{\ell}$ are sensitive to important cosmological parameters. The most robust constraint obtained is that on the spatial curvature of the Universe and baryon density. The observations establish that space on cosmic scales is geometrically flat $\left(\Omega_{K}=0\right)$ to within sub-percent precision. The dominant energy content in the present Universe is a mysterious matter with negative pressure dubbed, dark energy, or, the cosmological

${ }^{1}$ Wilkinson Microwave Anisotropy Probe mission http://wmap.gsfc.nasa.gov/ 
constant, which contributes about $73 \%$ of the total energy budget $\left(\Omega_{\Lambda}=0.73\right)$, followed by cold non-baryonic dark matter about $23 \%\left(\Omega_{m}=0.23\right)$ and, most humbly, ordinary matter (baryons) account for only about $4 \%\left(\Omega_{B}=0.04\right)$ of the matter budget. The current up to date status of cosmological parameter estimates from joint analysis of CMB anisotropy and large-scale structure (LSS) data is usually found in the parameter estimation paper accompanying the most recent results of a major experiment, such as the recent WMAP release of 7-year data (Komatsu et al. 2011; Larson et al. 2011).

More recently, $\mathrm{CMB}$ polarization measurements have provided the required complementary information on the nature of initial conditions for the primordial fluctuations. One of the firm predictions of the working 'standard' cosmological model is a random pattern of linear polarization ( $Q$ and $U$ Stokes parameters) imprinted on the CMB at last scattering surface. Thomson scattering generates CMB polarization anisotropy at decoupling (Bond \& Efstathiou 1984; Hu $\&$ White 1997). This arises from the polarization dependence of the differential cross section: $d \sigma / d \Omega \propto\left|\epsilon^{\prime} \cdot \epsilon\right|^{2}$, where $\epsilon$ and $\epsilon^{\prime}$ are the incoming and outgoing polarization states involving linear polarization only (Rybicki \& Lightman 1979). A local quadrupole temperature anisotropy produces a net polarization, because of the $\cos ^{2} \theta$ dependence of the cross section. A net pattern of linear polarization is retained due to local quadrupole intensity anisotropy of the CMB radiation impinging on the electrons at the last scattering surface. The polarization pattern on the sky can be decomposed in the two kinds with different parities. The even parity pattern arises as the gradient of a scalar field called the $E$-mode. The odd parity pattern arises from the 'curl' of a pseudo-scalar field called the $B$-mode of polarization. The observed CMB sky map is then characterized by a triplet of random scalar fields: $X(\hat{n}) \equiv\{\Delta T(\hat{n}), E(\hat{n}), B(\hat{n})\}$. It is possible to generalize equation (1) to express both $\mathrm{CMB}$ anisotropy and polarization in spherical harmonic space as

$$
X(\hat{\mathbf{n}})=\sum_{\ell=2}^{\infty} \sum_{m=-\ell}^{\ell} a_{\ell m}^{X} Y_{\ell m}(\hat{\mathbf{n}}) .
$$

and also define a set of observable angular power spectra analogous to eqn. (2) as

$$
C_{\ell}^{X X^{\prime}}=\frac{1}{(2 \ell+1)} \sum_{m=-\ell}^{\ell} a_{\ell m}^{X} a_{\ell m}^{X^{\prime *}} .
$$

For statistically isotropic, Gaussian CMB sky, there are a total of 4 power spectra that characterize the $\mathrm{CMB}$ signal : $C_{\ell}^{\mathrm{TT}}, C_{\ell}^{\mathrm{TE}}, C_{\ell}^{\mathrm{EE}}, C_{\ell}^{\mathrm{BB}}$. Parity conservation within standard radiative processes eliminates the two other possible power spectra, $C_{\ell}^{\mathrm{TB}} \& C_{\ell}^{\mathrm{EB}}$. Important point to note is that the odd-parity B-mode of polarization cannot sourced by scalar density perturbations, or potential velocity flow. B mode polarization can arise only due to shear fields acting on photon distribution, such as, from gravitational waves and (weak) gravitational lensing deflection of photons.

After the first detection of CMB polarization spectrum by the Degree Angular Scale Interferometer (DASI) on the intermediate band of angular scales $(l \sim 200-440)$ in late 2002 (Kovac et 
al. 2002), the field has rapidly grown, with measurements coming in from a host of ground-based and balloon-borne dedicated CMB polarization experiments. The full sky E-mode polarization maps and polarization spectra from WMAP were a new milestone in CMB research (Kogut et al. 2003; Page et al. 2007). Although the CMB polarization is a clean probe of the early Universe that promises to complement the remarkable successes of $\mathrm{CMB}$ anisotropy measurements it is also a much subtler signal than the anisotropy signal. Measurements of polarization by ongoing experiments at sensitivities of $\mu K$ (E-mode) have had to overcome numerous challenges in the past decade. The tens of $n K$ level B-mode signal pose the ultimate experimental and analysis challenge to this area of observational cosmology. The most current CMB polarization measurement of $C_{\ell}^{\mathrm{TT}}, C_{\ell}^{\mathrm{TE}}$ and $C_{\ell}^{\mathrm{EE}}$ and a non-detection of $B$-modes come from QUaD and BICEP. They also report interesting upper limits $C_{\ell}^{\mathrm{TB}}$ or $C_{\ell}^{\mathrm{EB}}$, over and above observational artifacts (Wu et al. 2009). A non-zero detection of $C_{\ell}^{\mathrm{TB}}$ or $C_{\ell}^{\mathrm{EB}}$, over and above observational artifacts, could be tell-tale signatures of exotic parity violating physics (Lue, Wang \& Kamionkowski 1999; Maity, Mazumdar \& Sengupta 2004) and the CMB measurements put interesting limits on these possibilities.

The immense dividends of CMB polarization measurements for understanding the physics behind the origin and evolution of our Universe have just started coming in. While CMB temperature anisotropy can also be generated during the propagation of the radiation from the last scattering surface, the CMB polarization signal can be generated primarily at the last scattering surface, where the optical depth of the Universe transits from large to small values. The polarization information complements the CMB temperature anisotropy by isolating the effect at the last scattering surface from other distinct physical effects acting during the propagation of the photons along the line of sight.

The polarization measurements provide an important test on the adiabatic nature of primordial scalar fluctuations 2. CMB polarization is sourced by the anisotropy of the CMB at recombination, consequently, the angular power spectra of temperature and polarization are closely linked. The power in the CMB polarization signal is sourced by the gradient (velocity) term in the same acoustic oscillations of the baryon-photon fluid at last scattering that gives rise to temperature (intensity) anisotropy. Hence, a clear evidence of adiabatic initial conditions for primordial fluctuations is that the compression and rarefaction peaks in the temperature anisotropy spectrum should be 'out of phase' with the gradient (velocity) driven peaks in the polarization spectra.

Figure 4 taken from Brown et al. (2009) reflects the current observational status of CMB E-mode polarization measurements. The recent measurements of the angular power spectrum the E-mode of CMB polarization at large $l$ have confirmed that the peaks in the $C_{\ell}^{\mathrm{EE}}$ spectra are out of phase with that of the temperature anisotropy spectrum $C_{\ell}^{\mathrm{TT}}$.

While the power in the CMB temperature anisotropy at low multipoles $(l \lesssim 60)$ first measured

\footnotetext{
${ }^{2}$ Another independent observational test comes from the recent measurements of the Baryon Acoustic Oscillations (BAO) in the power spectrum of LSS in the distribution of galaxies. BAO has also observationally established the gravitational instability mechanism for structure formation.
} 

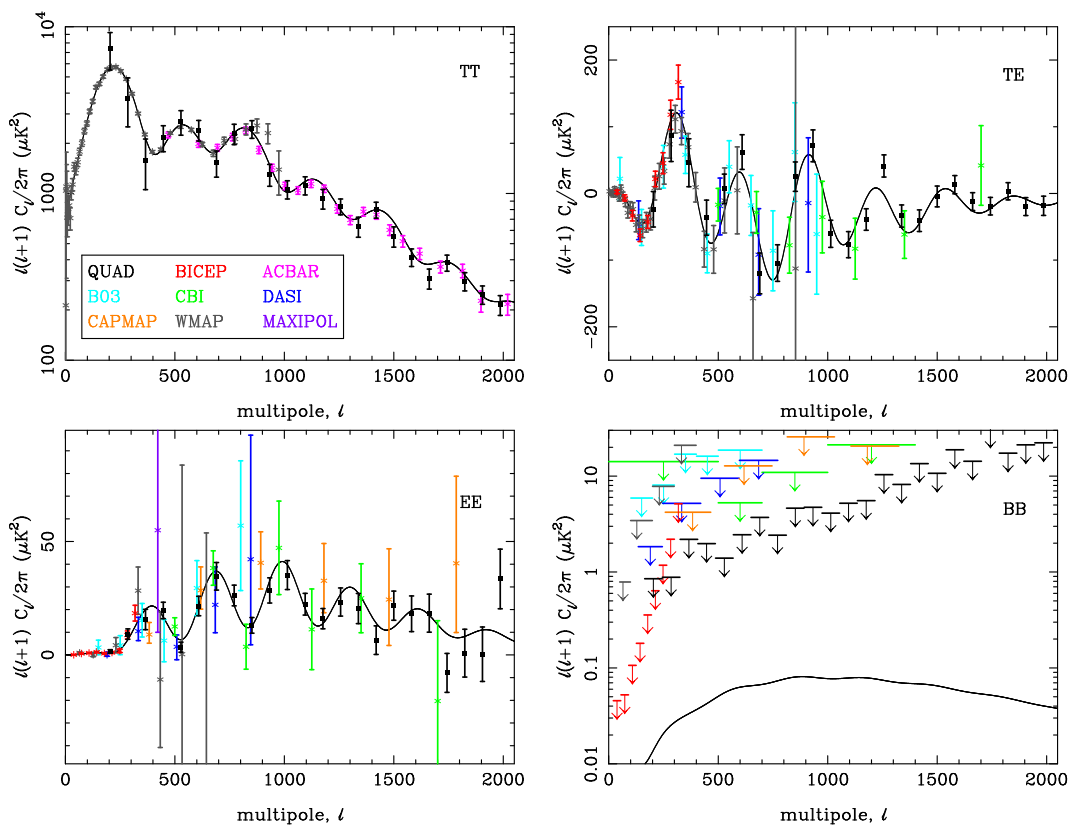

Figure 4. Figure taken from Brown et al. (2009) shows a compilation of recent measurements of the angular power spectra of CMB anisotropy and polarization from a number of CMB experiments. The data is good enough to indicate that the peaks in EE and TE are out of phase with that of TT as expected for adiabatic initial conditions. The null BB detection of primary CMB signal from gravity waves is not unexpected (given the ratio of tensor to scalar perturbations expected in the simplest models of inflation). It is important to note that the upper limits on $C_{\ell}^{\mathrm{BB}}$ have improved by almost an order of magnitude in the past two years from experiments such as QUAD and BICEP.

by the COBE-DMR (Smoot et al. 1992) did point to the existence of correlated cosmological perturbations on super Hubble-radius scales at the epoch of last scattering, it left open the (rather unlikely) 'logical' alternative possibility that all the power at low multipole is generated through integrated Sachs-Wolfe effect along the line of sight later in the Universe (when the Hubble scale is larger). However, since the polarization anisotropy is generated only at the last scattering surface, the negative trough clearly visible at high significance in the $C_{\ell}^{\mathrm{TE}}$ spectrum at $l \sim 130$ (that corresponds to a scale larger than the horizon at the epoch of last scattering) sealed this loophole, and provides an unambiguous proof of apparently 'acausal' correlations in the cosmological perturbations. This was first first measured by WMAP and later reconfirmed with higher significance by QUaD and BICEP (Kogut et al. 2003; Bennett et al. 2003; Page et al. 2007; Brown et al. 2009; Chiang et al. 2010).

The B-mode CMB polarization is a very clean and direct probe of the early Universe physics that generated the primordial metric perturbations. Inflationary models necessarily produce tensor perturbations (gravitational waves) that are predicted to evolve independently of the scalar 
density perturbations, with an uncorrelated power spectrum. The tensor modes on the scales of Hubble-radius the line of sight to the last scattering distort the photon propagation and generate an additional anisotropy pattern predominantly on the largest scales. (The amplitude of a tensor mode falls off rapidly on sub-Hubble radius scales, hence is important on angular scales comparable to larger than the Hubble radius at last scattering). It is common to parametrize the tensor component by the ratio $r_{k_{s}}=A_{\mathrm{t}} / A_{\mathrm{s}}$, ratio of $A_{\mathrm{t}}$, the primordial power in the transverse traceless part of the metric tensor perturbations, and $A_{\mathrm{s}}$, the amplitude scalar perturbation at a comoving wave-number, $k_{*}\left(\right.$ in $\mathrm{Mpc}^{-1}$ ). For power-law models, recent WMAP data alone puts an improved upper limit on the tensor to scalar ratio, $r_{0.002}<0.55(95 \% \mathrm{CL})$ and the combination of WMAP and the lensing-normalized SDSS galaxy survey implies $r_{0.002}<0.28$ (95\% CL) (MacTavish et al. 2006).

On angular scales corresponding to the multipole range $50<\ell<150$, the B (curl) component of CMB polarization is a unique signature of tensor perturbations from inflation. The amplitude of tensor perturbation is directly proportional to Hubble parameter during inflation, Loosely speaking, this is related to the Hawking temperature in de-Sitter like space-times. In turn, $H_{\text {inf }}$ is related to the energy density $\mathcal{E}_{\text {Inf }}$ of the Universe during inflation through the Friedman equation governing cosmological evolution. Hence, the CMB B-polarization is a direct probe of the energy scale of early Universe physics that generated the primordial metric perturbations (scalar \& tensor). The relative amplitude of tensor to scalar perturbations, $r$, sets the energy scale for inflation $\mathcal{E}_{\text {Inf }}=3.4 \times 10^{16} \mathrm{GeV} \mathrm{r}^{1 / 4}$. A measurement of $B$-mode polarization on large scales would give us this amplitude, and hence a direct determination of the energy scale of inflation. Besides being a generic prediction of inflation, the cosmological gravity wave background from inflation would be also be a fundamental test of GR on cosmic scales and the semi-classical behavior of gravity. Figure 5 summarizes the current theoretical understanding, observational constraints, and future possibilities for the stochastic gravity wave background from inflation. The stochastic gravitational wave background from inflation is expected to exist on cosmological scales down to terrestrial scales. The first CMB normalized GW spectra from inflation using the COBE results was given by Souradeep \& Sahni (1992) from IUCAA. This prediction will be targeted by both $\mathrm{CMB}$ polarization experiments, as well as, future $\mathrm{GW}$ observatories in space, such as Big Bang Observatory (BBO), DECIGO and LISA (Marx et al. 2010).

Gravitational lensing of the stronger CMB E-polarization by ongoing process of structures formation along the line of sight to the last scattering surface also generates a significant $B$ mode polarization, but on smaller angular scales $(\ell>200)$. This is prediction is shown as the black curve in bottom left panel for $C_{\ell}^{\mathrm{BB}}$ measurements in Fig. 4. The lensing signal carries important information about the matter power spectrum and its evolution over a range of redshift inaccessible to other observations. This promises a powerful probe for constraining the nature of dark energy and, also more excitingly, for determining the neutrino masses. Recent studies indicate that measuring the lensing polarization signal to the cosmic variance limit, can potentially place limits on the total mass of neutrinos at a level comparable to the measured mass differences from neutrinos oscillations.

While there has been no detection of cosmological signal in B-mode of polarization, the 


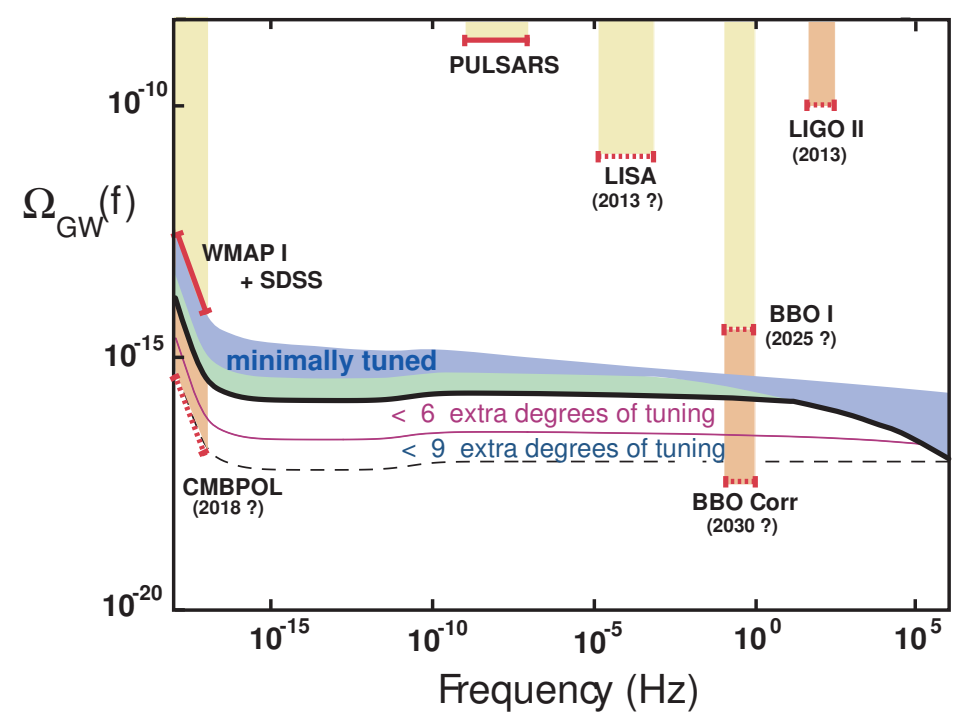

Figure 5. The figure taken from Boyle, Steinhardt \& Turok 2006, and NASA/DOE/NSF Task force report on Cosmic Microwave Background research, 2005 (http://www.nsf.gov/mps/ast/tfcr.jsp) shows the theoretical predictions and observational constraints on primordial gravitational waves from inflation. The gravitational wave energy density per logarithmic frequency interval, (in units of the critical density) is plotted versus frequency. The shaded (blue) band labeled 'minimally tuned' represents the range predicted for simple inflation models with the minimal number of parameters and tunings. The dashed curves have lower values of tensor contribution, $r$, that is possible with more fine tuned inflationary scenarios. The currently existing experimental constraints shown are due to: big bang nucleosynthesis (BBN), binary pulsars, and WMAP-1 (first year) with SDSS. Also shown are the projections for LIGO (both LIGO-I, after one year running, and LIGO-II); LISA; and BBO (both initial sensitivity, BBO-I, and after cross-correlating receivers, BBO-Corr). Also seen the projected sensitivity of a future space mission for CMB polarization (CMBPol).

lack of $B$-mode power suggests that foreground contamination from polarized emission from our own Galaxy is at a manageable level and is very encouraging news for the prospects of future measurements. The Planck satellite launched in May 2009 will greatly advance our knowledge of CMB polarization by providing foreground/cosmic variance-limited measurements of $C_{\ell}^{\mathrm{TE}}$ and $C_{\ell}^{\mathrm{EE}}$ out beyond $l \sim 1000$. We also expect to detect the weak lensing signal in $C_{\ell}^{\mathrm{BB}}$, although with relatively low precision, that required for placing ultimate limits on the total neutrino mass. Perhaps, Planck could also detect the stochastic inflationary gravitational wave background if it exists at a level of $r \sim 0.1$. Dedicated future CMB polarization space missions are under study at both NASA and ESA in the time frame 2020+3. Lower budget missions would primarily target

3 NASA/DOE/NSF Task force report on Cosmic Microwave Background research, 2005. http://www.nsf.gov/mps/ast/tfcr.jsp (Also available at the Legacy Archive for Microwave Background Data analysis (LAMBDA) site http://lambda.gsfc.nasa.gov). 
the low multipole $B$-mode polarization signature of gravity waves and consequently, identify the viable sectors in the space of inflationary parameters. More ambitious plans such proposal, such as COrE 4 , target the entire useful $C_{\ell}^{\mathrm{BB}}$ spectrum and also plan to probe other exciting results from $\mathrm{CMB}$ weak lensing measurements.

\section{Beyond the angular power spectra of the CMB sky}

It is well appreciated that in 'classical' big bang model the initial perturbations would have had to be generated 'acausally'. Besides resolving a number of other problems of classical Big Bang, inflation provides a mechanism for generating these apparently 'acausally' correlated primordial perturbations (Starobinsky 1982; Guth \& Pi 1982; Bardeen et al. 1983). There is increasing effort towards establishing this observationally. There are subtle observations of the CMB sky that could reveal more clearly the mechanism for generations of primordial fluctuation, or, perhaps surprise us by producing insurmountable challenges to the inflation paradigm.

\subsection{Statistical isotropy of the Universe}

The Cosmological Principle that led to the idealized FRW Universe found its strongest support in the discovery of the (nearly) isotropic, Planckian, cosmic microwave background. The isotropy around every observer leads to spatially homogeneous cosmological models. The large scale structure in the distribution of matter in the Universe (LSS) implies that the symmetries incorporated in FRW cosmological models ought to be interpreted statistically. These are also predicted in the simplest models of inflation.

The CMB anisotropy and its polarization is currently the most promising observational probe of the global spatial structure of the Universe on length scales close to, and even somewhat beyond, the 'horizon' scale $\left(\sim \mathrm{cH}_{0}^{-1}\right)$. The exquisite measurement of the temperature fluctuations in the CMB provide an excellent test bed for establishing the statistical isotropy (SI) and homogeneity of the Universe. In 'standard' cosmology, CMB anisotropy signal is expected to be statistically isotropic, i.e., statistical expectation values of the temperature fluctuations $\Delta T(\hat{q})$ are preserved under rotations of the sky. In particular, the angular correlation function $C\left(\hat{q}, \hat{q}^{\prime}\right) \equiv\left\langle\Delta T(\hat{q}) \Delta T\left(\hat{q}^{\prime}\right)\right\rangle$ is rotationally invariant for Gaussian fields. In spherical harmonic space, where $\Delta T(\hat{q})=\sum_{l m} a_{l m} Y_{l m}(\hat{q})$, the condition of statistical isotropy (SI) translates to a diagonal $\left\langle a_{l m} a_{l^{\prime} m^{\prime}}^{*}\right\rangle=C_{l} \delta_{l l^{\prime}} \delta_{m m^{\prime}}$ where $C_{l}$, is the widely used angular power spectrum of CMB anisotropy. The $C_{l}$ is a complete description only of (Gaussian) SI CMB sky CMB anisotropy and would be (in principle) an inadequate measure for comparing models when SI is violated (Bond, Pogosyan \& Souradeep 1998, 2000a,b).

Interestingly enough, the statistical isotropy of CMB has come under a lot of scrutiny after

${ }^{4}$ Cosmic Origins Explorer (COrE) proposal, http://www.core-mission.org 
the WMAP results. Tantalizing evidence of SI breakdown (albeit, in very different guises) has mounted in the WMAP first year sky maps, using a variety of different statistics. It was pointed out that the suppression of power in the quadrupole and octupole are aligned (Tegmark, de OliveiraCosta \& Hamilton 2004). Further "multipole-vector" directions associated with these multipoles (and some other low multipoles as well) appear to be anomalously correlated (Copi, Huterer \& Starkman 2004; Schwartz et al. 2004). There are indications of asymmetry in the power spectrum at low multipoles in opposite hemispheres (Eriksen et al. 2004). Analysis of the distribution of extrema in WMAP sky maps has indicated non-Gaussianity, and to some extent, violation of SI (Larson \& Wandelt 2004). The more recent WMAP maps are consistent with the first-year maps up to a small quadrupole difference. The additional years of data and the improvements in analysis has not significantly altered the low multipole structures in the maps (Hinshaw et al. 2007). Hence, 'anomalies' persisted at the same modest level of significance and are unlikely to be artifacts of noise, systematics, or the analysis in the first year data. The cosmic significance of these 'anomalies' remains debatable also because of the aposteriori statistics employed to ferret them out of the data. The WMAP team has devoted an entire publication to discuss and present a detailed analysis of the various anomalies (Bennett et al. 2011).

The observed CMB sky is a single realization of the underlying correlation, hence detection of SI violation, or correlation patterns, pose a great observational challenge. It is essential to develop a well defined, mathematical language to quantify SI and the ability to ascribe statistical significance to the anomalies unambiguously. The Bipolar spherical harmonic (BipoSH) representation of $\mathrm{CMB}$ correlations has proved to be a promising avenue to characterize and quantify violation of statistical isotropy.

Two point correlations of CMB anisotropy, $C\left(\hat{n}_{1}, \hat{n}_{2}\right)$, are functions on $S^{2} \times S^{2}$, and hence can be generally expanded as

$$
C\left(\hat{n}_{1}, \hat{n}_{2}\right)=\sum_{l_{1}, l_{2}, \ell, M} A_{l_{1} l_{2}}^{\ell M} Y_{\ell M}^{l_{1} l_{2}}\left(\hat{n}_{1}, \hat{n}_{2}\right)
$$

Here $A_{l_{1} l_{2}}^{\ell M}$ are the Bipolar Spherical harmonic (BipoSH) coefficients of the expansion and $Y_{\ell M}^{l_{1} l_{2}}\left(\hat{n}_{1}, \hat{n}_{2}\right)$ are bipolar spherical harmonics. Bipolar spherical harmonics form an orthonormal basis on $S^{2} \times S^{2}$ and transform in the same manner as the spherical harmonic function with $\ell, M$ with respect to rotations. Consequently, inverse-transform of $C\left(\hat{n}_{1}, \hat{n}_{2}\right)$ in eq. (5) to obtain the BipoSH coefficients of expansion is unambiguous.

Most importantly, the Bipolar Spherical Harmonic (BipoSH) coefficients, $A_{l_{1} l_{2}}^{\ell M}$, are linear combinations of off-diagonal elements of the harmonic space covariance matrix,

$$
A_{l_{1} l_{2}}^{\ell M}=\sum_{m_{1} m_{2}}\left\langle a_{l_{1} m_{1}} a_{l_{2} m_{2}}^{*}\right\rangle(-1)^{m_{2}} C_{l_{1} m_{1} l_{2}-m_{2}}^{\ell M}
$$

where $C_{l_{1} m_{1} l_{2} m_{2}}^{\ell M}$ are Clebsch-Gordan coefficients and completely represent the information of the covariance matrix.

Statistical isotropy implies that the covariance matrix is diagonal, $\left\langle a_{l m} a_{l^{\prime} m^{\prime}}^{*}\right\rangle=C_{l} \delta_{l l^{\prime}} \delta_{m m^{\prime}}$ 
and hence the angular power spectra carry all information of the field. When statistical isotropy holds BipoSH coefficients, $A_{l^{\prime}}^{\ell M}$, are zero except those with $\ell=0, M=0$ which are equal to the angular power spectra up to a $(-1)^{l}(2 l+1)^{1 / 2}$ factor. Therefore to test a CMB map for statistical isotropy, one should compute the BipoSH coefficients for the maps and look for nonzero BipoSH coefficients. Statistically significant deviations of BipoSH coefficient of map from zero would establish violation of statistical isotropy.

Since $A_{l_{1} l_{2}}^{\ell M}$ form an equivalent representation of a general two point correlation function, cosmic variance precludes measurement of every individual $A_{l_{1} l_{2}}^{\ell M}$. There are several ways of combining BipoSH coefficients into different observable quantities that serve to highlight different aspects of SI violations. Among the several possible combinations of BipoSH coefficients, the Bipolar Power Spectrum (BiPS) has proved to be a useful tool with interesting features (Hajian \& Souradeep 2003; Hajian, Souradeep \& Cornish 2005). BiPS of CMB anisotropy is defined as a convenient contraction of the BipoSH coefficients

$$
\kappa_{\ell}=\sum_{l, l^{\prime}, M} W_{l} W_{l^{\prime}}\left|A_{l l^{\prime}}^{\ell M}\right|^{2} \geq 0
$$

where $W_{l}$ is the window function that corresponds to smoothing the map in real space by symmetric kernel to target specific regions of the multipole space and isolate the SI violation on corresponding angular scales.

The BipoSH coefficients can be summed over $l$ and $l^{\prime}$ to reduce the cosmic variance, to as obtain reduced BipoSH (rBipoSH) coefficients (Hajian \& Souradeep 2006)

$$
A_{\ell M}=\sum_{l=0}^{\infty} \sum_{l^{\prime}=|\ell-l|}^{\ell+l} A_{l l^{\prime}}^{\ell M}
$$

Reduced bipolar coefficients orientation information of the correlation patterns. An interesting way of visualizing these coefficients is to make a Bipolar map from $A_{\ell M}$

$$
\Theta(\hat{n})=\sum_{\ell=0}^{\infty} \sum_{M=-\ell}^{\ell} A_{\ell M} Y_{\ell M}(\hat{n})
$$

The symmetry $A_{\ell M}=(-1)^{M} A_{\ell-M}^{*}$ of reduced bipolar coefficients guarantees reality of $\Theta(\hat{n})$.

It is also possible to obtain a measurable band power measure of $A_{l_{1} l_{2}}^{\ell M}$ coefficient by averaging $l_{1}$ in bands in multipole space. Recently, the WMAP team has chosen to quantify SI violation in the CMB anisotropy maps by the estimation $A_{l l-i}^{\ell M}$ for small value of bipolar multipole, $L$, band averaged in multipole $l$. Fig. 3.1 taken from the WMAP-7 release paper (Bennett et al. 2011) shows SI violation measured in WMAP CMB maps

CMB polarization maps over large areas of the sky have been recently delivered by experiments in the near future. The statistical isotropy of the CMB polarization maps will be an independent probe of the cosmological principle. Since CMB polarization is generated at the surface 


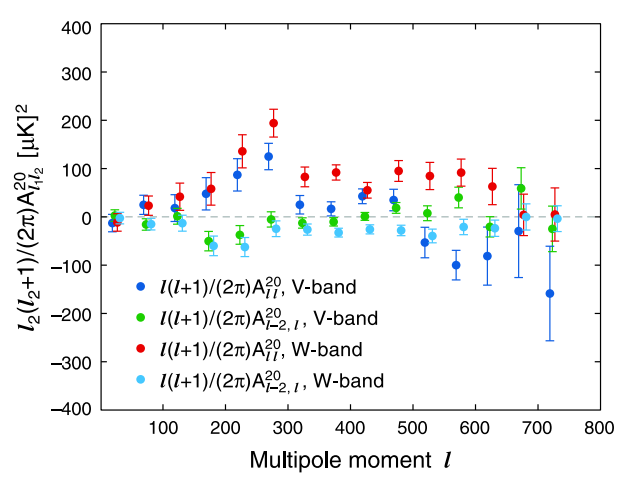

Figure 6. Figure taken from WMAP-7 yr publication on anomalies in the CMB sky (Bennett et al. 2011) shows the measured quadrupolar (bipolar index $L=2$ ) bipolar power spectra for V-band and W-band WMAP data, using the KQ75y7 mask. The spherical multipole have been binned within uniform bands $\delta l=50$. Only the components of the bipolar power spectra with $\mathrm{M}=0$ in ecliptic coordinates are shown. A statistically significant quadrupolar effect is seen, even for a single frequency band in a single angular bin.

of last scattering, violations of statistical isotropy are pristine cosmic signatures and more difficult to attribute to the local Universe. The Bipolar Power spectrum has been defined and implemented for CMB polarization and show great promise (Basak, Hajian \& Souradeep 2006; Souradeep, Hajian \& Basak 2006).

\subsection{Gaussian primordial perturbations}

The detection of primordial non-Gaussian fluctuations in the CMB would have a profound impact on our understanding of the physics of the early Universe. The Gaussianity of the CMB anisotropy on large angular scales directly implies Gaussian primordial perturbations (Munshi, Souradeep \& Starobinsky 1995; Spergel \& Goldberg 1999) that is theoretically motivated by inflation. The simplest inflationary models predict only very mild non-Gaussianity that should be undetectable in the WMAP data.

The CMB anisotropy maps (including the non Gaussianity analysis carried out by the WMAP team data; Komatsu et al. 2011) have been found to be consistent with a Gaussian random field. Consistent with the predictions of simple inflationary theories, no significant deviations from Gaussianity in the CMB maps using general tests such as Minkowski functionals, the bispectrum, trispectrum in the three year WMAP data (Spergel et al. 2007; Komatsu et al. 2011). There have however been numerous claims of anomalies in specific forms of non-Gaussian signals in the CMB data from WMAP at large scales (see discussion in sec. 3.1). Recently, a new class of odd-parity bispectra has been discovered enriching the field significantly (Kamionkowski \& Souradeep 2011). 


\section{Summary}

The past few years has seen the emergence of a 'concordant' cosmological model that is consistent both with observational constraints from the background evolution of the Universe as well that from the formation of large scale structures. It is certainly fair to say that the present edifice of the 'standard' cosmological models is robust. A set of foundation and pillars of cosmology have emerged and are each supported by a number of distinct observations (Ostriker \& Souradeep 2004; Souradeep 2011).

Besides precise determination of various parameters of the 'standard' cosmological model, observations have also established some important basic tenets of cosmology and structure formation in the Universe - 'acausally' correlated initial perturbations, adiabatic nature primordial density perturbations, and gravitational instability as the mechanism for structure formation. The favored, concordance model inferred is a spatially flat accelerating Universe where structures have formed by the gravitational evolution of nearly scale invariant, adiabatic perturbations, as expected from inflation. The signature of primordial perturbations observed through the CMB anisotropy and polarization is the most compelling evidence for new, possibly fundamental, physics in the early Universe that underlie the scenario of inflation (or related alternatives). Searches are also on for subtle signals in the CMB maps beyond the angular power spectrum in the violation of statistical isotropy (Hajian \& Souradeep 2003, 2006; Hajian, Souradeep \& Cornish 2005; Basak, Hajian \& Souradeep 2006; Souradeep, Hajian \& Basak 2006), or, in the violation of Gaussianity (Munshi, Souradeep \& Starobinksy 1995; Spergel \& Goldberg 1999).

Cosmology is a branch of physics that has seen theoretical enterprise at its best. During the long period of sparse observations in its history, brilliant theoretical ideas (and prejudices) shaped a plausible self-consistent scenario. But current cosmology is passing through a revolution. In the recent past, cosmology has emerged as a data rich field increasingly driven by exquisite and grand observations of unprecedented quality and quantity. These observations have transformed cosmology into an emergent precision science of this century. Further, CMB polarization is arguably emerging as a key observable that can also address fundamental questions related to the origin of the Universe.

\section{Acknowledgments}

I would like to thank the editors, D. J. Saikia and Virginia Trimble for inviting me to write this article and for their immense patience in obtaining it from me. It is indeed a privilege to contribute to a commemorative volume honoring an iconic scientist, Prof. S. Chandrasekhar, whom I had the good fortune to meet, and talk to, early in my research career at IUCAA. Finally, I acknowledge my past and present students and collaborators who have contributed to my own research in this exciting area. 


\section{References}

Bardeen J.M., Steinhardt P.J., Turner M.S., 1983, Phys. Rev. D, 28, 679

Basak S., Hajian A., Souradeep T., 2006, Phys. Rev. D, 74, 021301(R)

Bennett C.L., et al., 2003, ApJS, 148, 1

Bennett, C., et al., 2011, ApJS, 192, 17

Bond J.R. 1996, in Schaeffer R., ed, Cosmology and Large Scale Structure, Les Houches Session LX, August 1993, Elsevier Science Press

Bond J.R., Efstathiou G., 1984, ApJ 285, L45

Bond J.R., Efstathiou G., 1987, MNRAS, 226, 655.

Bond J.R., Pogosyan D., Souradeep T., 1998, Class. Quant. Grav., 15, 2671

Bond J.R., Pogosyan D., Souradeep T., 2000a, Phys. Rev. D, 62, 043005

Bond J.R., Pogosyan D., Souradeep T., 2000b, Phys. Rev. D, 62, 043006

Boyle L.A., Steinhardt P.J., Turok N., 2006, Phys. Rev. Lett., 96, 111301

Brown M.L., et al., 2009, ApJ, 2009, 705, 798

Chandrasekhar S., 1960, Radiative transfer, Dover publications, New York

Chiang H.C., et al., 2010, ApJ, 711, 1123

Copi C.J., Huterer D., Starkman G.D., 2004, Phys. Rev. D, 70, 043515

Eriksen H. K., et al., 2004, ApJ, 605, 14

Guth A.H., Pi S.-Y., 1982, Phys. Rev. Lett., 49, 1110

Hajian A., Souradeep T., 2003, ApJL, 597, L5

Hajian A., Souradeep T., 2006, Phys.Rev., D74, 123521

Hajian A., Souradeep T., Cornish N., 2005, ApJL, 618, L63

Hinshaw G., et al., 2007, ApJS, 170, 288

Hu W., Dodelson S., 2002, ARA\&A, 40, 171

Hu W., White M., 1997, New Astron., 2, 323

Hu W., Sugiyama N., Silk J., 1997, Nature, 386, 37

Kamionkowski M., Souradeep T., 2011, Phys. Rev. D, 83, 027301

Kogut A., et. al., 2003, ApJS., 148, 161

Komatsu E., et al., 2011 ApJS, 192, 18

Kovac J. M., et al.,2002 Nature, 420, 772

Larson D., et.al., 2011 ApJS, 192, 16

Larson D.L., Wandelt B.D., 2004, ApJL, 613, L85

Lue A., Wang L., Kamionkowski M., 1999, Phys. Rev. Lett., 83, 1506

MacTavish C.J., et al., 2006, ApJ, 647, 799

Maity D., Majumdar P., Sengupta S., 2004, JCAP, 0406, 005

Marx J., et al., 2010, GWIC Roadmap document, https://gwic.ligo.org/roadmap/

Munshi D., Souradeep T., Starobinsky A., 1995, ApJ, 454, 552

Ostriker J.P., Souradeep T., 2004, Pramana, 63, 817

Page L., et al., 2007, ApJS, 170, 335

Peebles P.J.E., Yu J.T.,1970, ApJ, 162, 815

Rybicki G.B., Lightman A.P., 1979, Radiative processes in astrophysics, New York: Wiley-Interscience

Saha R., Jain P., Souradeep T., 2006, ApJL, 645, L89

Samal P., et al., 2010, ApJ, 714, 840

Schwarz D.J., et al., 2004, Phys. Rev. Lett., 93, 221301

Smoot G.F., et al., 1992, ApJL, 396, L1

Souradeep T., 2011, in Proc. GR-19 July 2010,Mexico, eds, Marolf D., Sudarsky D., Class. Q. Grav., in press 
Souradeep T., Sahni V., 1992, Mod. Phys. Lett. A, 7, 3541

Souradeep T., Hajian A.,Basak S., 2006, New Astron.Rev., 50, 889

Spergel D.N., Goldberg D.M., 1999, Phys. Rev. D, 59, 103001

Spergel D., et al., 2007, ApJS, 170, 377

Starobinsky A. A., 1982, Phys. Lett., 117B, 175

Tegmark M., de Oliveira-Costa A., Hamilton A., 2004, Phys. Rev. D, 68, 123523

Wu E.Y.S., et al., 2009, Phys.Rev.Lett., 102, 161302 